\title{
Connecting Brain and Heart: Artificial Intelligence for Sustainable Development
}

\author{
Diego Chavarro ${ }^{1}$, Jaime Andrés Pérez-Taborda ${ }^{2}$, and Alba Ávila ${ }^{3 *}$ \\ ${ }^{1}$ dchavarro@gmail.com \\ Colombian Society of Engineering Physics (SCIF), 660003, Pereira (Colombia) \\ 2jperezta@unal.edu.co \\ Colombian Society of Engineering Physics (SCIF), 660003, Pereira (Colombia)
}

Universidad Nacional de Colombia Sede de La Paz, Escuela de pregrados-Dirección Académica, Grupo de

Nanoestructuras y Física Aplicada (NANOUPAR) Km 9 vía Valledupar La Paz, La Paz, 202010. Colombia

${ }^{3}$ a-avila@uniandes.edu.co

Microelectronics Center (CMUA), Department of Electrical and Electronic Engineering, Universidad de Los Andes, 111711, Bogota (Colombia)

Colombian Society of Engineering Physics (SCIF), 660003, Pereira (Colombia)

*Corresponding author

\section{Note: this is a preprint. The final reviewed version will be available in the journal Scientometrics.}

\begin{abstract}
A key objective of global policies on Artificial Intelligence (AI) is to foster AI research for sustainable development (SD). In this paper, we analyze the inclusion of SD in AI research indexed by the IEEE Xplore database from 2000 to 2019. We address three critical questions: 1) To what extent is AI research addressing the sustainable development goals (SDGs)? 2) Which subject areas of AI show an emerging interest in SD? And 3) What patterns of collaboration between regions of the world are being stimulated by AI? Our scientometric analysis consists of 1) Identifying the number of AI papers that address SDGs in their titles, abstracts, and keywords. 2) Developing a composite indicator based on the number of documents produced, scientific impact, and inventive impact to distinguish areas with an emerging interest in SD; 3) Exploring coauthorship networks at three levels: region, income group, and country. The overall results show that a small share of papers is explicitly focused on SD. Our composite indicator allowed us to identify an emerging interest in SD from Ultrasonics, Ferroelectrics, and Frequency Control, Education, Consumer Electronics, Electrical Engineering, Electromagnetic Compatibility and Interference. Specifically, on AI subjects, we found emerging interests in Prediction Methods, Computation Theory, Machine Learning, Learning (artificial intelligence), and Biological Neural Networks. Inter-regional and inter-income group collaboration are limited, and network power is concentrated in a few countries. The results could be useful to improve the connection between technical knowledge, strategic planning for S\&T investment, and SD policies.
\end{abstract}

Keywords: Artificial Intelligence; Sustainable Development; Engineering and Society; Research Policy; Emerging Technologies

JEL codes: 031; 030; 033; 038; 057

\section{Acknowledgments}

This paper substantially extends the proceedings paper presented at the ISSI 2021 conference (Chavarro, PerezTaborda \& Avila 2021). We thank the IEEE for offering their open API, OSDGs for their web service to classify papers through machine learning, especially to Gusté Statulevičiūte and Lukas Pukelis from PPMI. We also thank the Free and Open-source software community: MariaDB, R, Gephi, and Notepad++ teams for making available their software. Special thanks to an anonymous reviewer and to Professor Wolfgang Glänzel for their excellent suggestions. 


\section{Introduction}

The fourth industrial revolution is characterized by the exponential development of technologies to radically transform life on Earth (Schwab 2017). This new revolution has been advocated as an opportunity to improve business productivity, automate various manual trades, provide greater control over the quality of production, among others (Mou 2019). On the other hand, there is growing concern about the adverse effects of this revolution on the environment, the economy, and society (Harari 2017). The transformations that the fourth industrial revolution is having in the world make it important to understand its risks and potential benefits, and the role of academia, industry, government, and civil society in steering it.

From a global perspective, there is a competition for leadership in the fourth industrial revolution's determining technologies. For example, most OECD member countries are improving their digital infrastructure, educating their citizens in information technologies, supporting new technology-based companies, and research in "convergent" and "exponential" technologies (Planes-Satorra \& Paunov 2019). The confrontation between China and the United States over the development and control of 5G technology (Sørensen et al. 2016) is an extreme but revealing example of the global competition situation. Consequently, several national and international agencies have begun to carry out policy studies to understand how to insert themselves into the new global value chain that is shaping the fourth industrial revolution and what international cooperation strategies can be more effective to position themselves as leaders in the technologies that drive this revolution (Kagermann et al. 2016; UNIDO 2018; Navarro 2018).

At the same time, a more cooperative agenda for science, technology, and innovation (STI) is being fostered by the global Sustainable Development Goals (SDGs) (Chavarro et al. 2017). The SDGs are a set of 17 social, economic, and environmental goals and 169 targets to be achieved by 2030, agreed upon by the 193 UN country members (UN 2021). STI plays a key role in achieving these goals by developing new knowledge for sustainable development, providing evidence for policymaking, creating scientific and technological capacity to cope with grand challenges, strengthening democratic practices, providing a critical perspective on issues such as the implementation of the agenda, monitoring progress in achieving the targets, among others (UNESCO 2015; Chavarro et al. 2017).

Since the fourth industrial revolution and the SDGs agendas co-exist, interact, and are incorporated into plans and policies of different stakeholders, one can explore the degree of "natural" connections that emerge between STI, the fourth industrial revolution, and the collaborative actions to achieve the 17 goals of Agenda 2030. A key question is if technological breakthroughs will work towards planetary well-being. If so, it is imperative to reconcile technological advancement with societal purposes (Cassi et al. 2017). Researchers have an essential role in achieving this reconciliation because they produce a good part of the enabling knowledge behind technology, and it is common that they produce the technologies themselves (Breschi et al. 2005; Zellmer-Bruhn et al. 2021).

Technologies are often seen as mere "technical" developments, but they also involve social aspects that need to be reflected upon by engineers (European Parliament 2020). For instance, AI algorithms to detect potential criminals can be biased by human assumptions on the physical appearance of people or their gender (Osoba \& Welser 2017). Therefore, reflecting beyond the technical aspects of technology is needed to produce humanistic technologies. This demands engineers to think of technologies to exert a positive impact on the planet because, as Jane Goodall puts it, "only when our clever brain and our human heart work together in harmony can we achieve our true potential" (Goodall 2014).

Part of achieving the true potential that Goodall talks about implies producing sustainable technologies with a clear purpose to ensure sustainable development (SD). Therefore, it is 
crucial to understand to what extent engineering research, which is behind the significant breakthroughs in world technology, addresses SD. In this paper, we understand SD as a development that aims to achieve a social, environmental, and economic balance (Chavarro et al., 2017).

We focus on $\mathrm{AI}$ as a ground-breaking domain of technologies and research with the power to redefine global society. Recently, the OECD issued a crucial policy paper in which they recognize that "AI should benefit people and the planet by driving inclusive growth, sustainable development and well-being" (OECD 2020). The IEEE has also made available a clear position to "support the R\&D needed to advance innovation and development in artificial intelligence and its application to benefit humanity" (IEEE 2019). Our work is a contribution to a broad question, which remains unexplored, on the extent to which "the digital era could contribute to or jeopardize sustainable landscapes (...), what is the role of digital technologies in pursuing the (2030) Agenda and how to enable a sustainable digitalization" (del Río Castro et al. 2020). Within this context, we explore the following questions:

1. To what extent is SD addressed in AI research produced globally?

2. Which subject areas of AI show an emerging interest in SD?

3. To what extent does AI stimulate collaboration between regions of the world?

We adopt a "bottom-up" approach by identifying what is already happening to achieve both technological advancement and planetary health. We look at the publications on AI available through the Institute of Electrical and Electronics Engineers - IEEE Xplore. Investigating AI from this perspective may help formulate evidence-based public policies to foster the developments that are already taking place, better understand the disciplinary map that is being configured in the world of AI and explore the extent to which the intellectual work of engineering researchers is considering the wider uses of their technologies and their impacts on the environment and society. This paper builds on the proceedings paper presented at the ISSI 2021 conference (Chavarro, Perez-Taborda \& Avila 2021), adding a more in-depth analysis of the core categories of AI research in the IEEE taxonomy. Our work could be useful to align the connection between technical knowledge, strategic planning for S\&T investment, and SD policies. Furthermore, our composite indicator can be used to measure the SD orientation of AI research areas for funding, complementing efforts to identify the AI content of research (Yamashita et al. 2021) that do not consider its directionality.

\section{Methodology}

This paper focuses on AI as an enabling technology (European Patent Office 2020) that will experience exceptional growth over the next years (Klavans, Boyack, \& Murdick 2020). AI is also one of the subjects for which there will be sustained policy support (European Commission 2020).

The term AI has gained thrust in the academic and policy domains (see Nilson 2010; IEEE 2019), but its definition remains contested and vague. Considering the two concepts "artificial" and "intelligence", a significant challenge is to understand their meaning. Some propose not one but multiple intelligences, such as Gardner, who introduces eight types of intelligence through their Multiple Intelligences Theory (Gardner 2000). What type of intelligence is expected in AI? Regardless of the definition of intelligence, what is programmed into a machine is a human's understanding of it, not yet a machine's intelligence. Moreover, if artificial refers to machine, what should be considered a machine? A pure non-organic unit or a hybrid? 
The definition challenge remains when analyzing the terms artificial and intelligence in combination. Simmons and Chappell (1988) discuss different understandings that range from mimicking the behavior of humans by machines to machines with minds of their own, and Russell (2010, p. 5) finds at least four domains of AI that cover the range of inventions and research on the subject. These are: acting like a human, thinking like a human, acting rationally, and thinking rationally. Other approaches even go beyond human intelligence, such as the developers of an algorithm that can defeat the best players of Go who consider that a goal of AI is to achieve "superhuman proficiency in challenging domains" (Silver et al., 2017, p.354). Because of the difficulties to define AI, Simmons \& Chappell (1988, p.15), prefer to refer to these technologies as "knowledge engineering, and the systems created as knowledge-based systems". This definition, however, does not remove the challenge in defining "knowledge" and "knowledge-based".

Another way to understand the concept of AI is by looking at how it has been adopted by different organizations and purposes. Some organizations, such as the International Organization for Standardization (ISO), do not provide a literal definition of AI and instead prefer to identify the components of a mapped AI ecosystem of technologies (Samoili et al., 2021, p. 9). Others, such as the European Commission, define AI by a set of identified "techniques and approaches" (European Commission, 2021, p. 39). The IEEE (2021a) is developing standard AI definitions for different technological niches and social domains, which suggests that the definition of AI is relative to its users and producers.

What emerges from the above remarks and a review of 65 different definitions of AI conducted by Samoili et al. (2021) is that defining AI "falsely gives the impression of a precision that cannot be obtained" (p.9). Due to the multiplicity of conceptualizations, we do not attempt a definition of AI. Instead, we identify AI documents by searching for the keywords "artificial intelligence" in titles, abstracts, and keywords of research papers in the Engineering domain from 2000 to 2020 in the IEEE Xplore database. This allows for different definitions to be included in our search. Besides, we use the classification of the IEEE thesaurus for individual papers (IEEE 2021a), which uses the criteria of indexers from an authoritative source for different Engineering subjects, including AI (IEEE 2021a).

\section{Data}

Our sample consists of documents developed in the Engineering Sciences, produced from 2000 to 2020 and indexed by the IEEE Xplore database with the keywords "artificial intelligence" in the fields title, abstract, and keywords, or at least one of the subjects being Artificial Intelligence (AI) (IEEE 2021a). Our choice has to do with the availability of finegrained classification of papers within AI that IEEE Xplore provides, which is required to produce meaningful analysis for experts in the discipline. Other more general citation databases could offer better interdisciplinary coverage, but the subject classifications may belong to non-Engineering disciplines studying AI. We are also aware that there might be biases towards the English language (Chavarro, Ràfols, and Tang 2018) and subjects that may be of more interest to high-income countries (Chavarro, Tang, and Ràfols 2017), issues that merit further research. 
In total, we have acquired 220,147 records of research articles, reviews, proceedings, and books. Table 1 shows the number of documents gathered per year, and Table 2 shows the number of documents by type of publication ${ }^{1}$ :

Table 1. Number of documents on AI per year

\begin{tabular}{rrcc}
\hline \multicolumn{1}{l}{$\begin{array}{l}\text { Publication } \\
\text { year }\end{array}$} & $\begin{array}{l}\text { Number of } \\
\text { documents }\end{array}$ & $\begin{array}{l}\text { Publication } \\
\text { year }\end{array}$ & $\begin{array}{l}\text { Number of } \\
\text { documents }\end{array}$ \\
\hline 2020 & 27,783 & 2009 & 8,606 \\
2019 & 39,269 & 2008 & 7,585 \\
2018 & 25,800 & 2007 & 6,022 \\
2017 & 17,503 & 2006 & 4,961 \\
2016 & 11,000 & 2005 & 4,552 \\
2015 & 9,797 & 2004 & 3,539 \\
2014 & 8,736 & 2003 & 2,740 \\
2013 & 8,064 & 2002 & 2,458 \\
2012 & 8,747 & 2001 & 1,805 \\
2011 & 9,591 & 2000 & 2,737 \\
2010 & 8,852 & & \\
\hline
\end{tabular}

Table 2. list of documents by type

\begin{tabular}{lr}
\hline Content-Type & $\begin{array}{c}\text { Number of } \\
\text { Documents }\end{array}$ \\
\hline Conference proceeding & 174,694 \\
Journal papers & 40,509 \\
Magazine papers & 2,959 \\
Early access articles & 1,291 \\
Books & 634 \\
Standards & 38 \\
Courses & 20 \\
\hline
\end{tabular}

\section{Data classification}

Two types of classifications were fundamental to understanding the relationship between sustainable development and AI in engineering. The first type is a subject classification, for which the IEEE Thesaurus was used. IEEE has also made available a taxonomy aimed explicitly at describing engineering subjects (IEEE 2021b). The IEEE taxonomy is derived from the IEEE Thesaurus, which is a controlled vocabulary of 10,644 terms. The taxonomy provides a hierarchical classification that represents the relationship between terms. As expert committees develop the Thesaurus and the taxonomy, they integrate expert knowledge and present data in a meaningful context to engineering research communities. Although this paper is about AI, we used the whole IEEE Taxonomy with its 52 categories (IEEE 2021b) because AI papers apply different engineering knowledge fields. For each paper, we identified its root term, first, second, and third levels according to the taxonomy (IEEE 2021b).

A critical aspect in our analysis is our second classification of papers into related and unrelated to SD, which remains a challenging task (Armitage, Lorenz, \& Mikki 2020)

\footnotetext{
${ }^{1}$ The search was performed over all publication fields in the database, using "artificial intelligence". The data was obtained through the IEEE Xplore API using PhP.
} 
because SD is not a disciplinary field, but a domain of interest linked to a long-term policy agenda, with a clear direction towards a better balance between human existence and the environment, economy, and society (Chavarro et al. 2017). Two main approaches have been developed by researchers for this task: the first is based on keyword searches curated by experts in the field (Elsevier 2020). This approach offers textual identification of elements related to sustainable development, but not semantic identification of concepts. The second approach uses Machine Learning (ML) algorithms to classify papers based on their similarity to a set of papers classified by humans (OSDG 2021). The latter offers a conceptual identification of papers. See table 3 for a comparison of the two approaches.

As both models have pros and cons, we combined the two classification strategies in this paper. For the keyword classification, we used Elsevier's keywords. For the machine learning approach, we used OSDG's (OSDG 2021), which is an initiative to provide a service for text classification into the categories of sustainable development goals. OSDG approach uses Microsoft Academic Knowledge Project, human annotation, and other resources as a means for topic classification (OSDG 2021). In total, the keyword approach produced 44,563 papers compared to 84,309 produced by the ML approach. We examined the results of the two approaches, following the advice by Ràfols et al. (2021), and found that the keyword approach offered classifications more consistent with our understanding of sustainable development in a random set of 1,000 papers checked manually by the team for this dataset.

Table 3. Assessment of SDG classification

\begin{tabular}{llllll}
\hline Method & Accuracy & Kappa & Precision & Recall & F-Scores \\
\hline Keyword & Raw: 74.5\% (95\% CI: 72\% & 0.36 & $68 \%$ & $42 \%$ & F0.5: 0.6055 \\
& $77 \%)$ & & & & F1: 0.5234 \\
& Balanced: 66\% & & & & F2: 0.4608 \\
ML & Raw: 67\% (95\% CI 64\%-69\%) & 0.23 & $49 \%$ & $48 \%$ & F0.5: 0.4876 \\
& Balanced: 62\% & & & F1: 0.4854 \\
& & & & F2: 0.4831 \\
\hline
\end{tabular}

The measures in table 3 are used in information science to indicate the robustness of a classification test (Powers 2020). These measures operate on a contingency table with four cells. 1) True Positives (TP): number of cases that are correctly predicted by the algorithm as pertaining to the category of interest; 2) True Negatives (TN): number of cases correctly predicted as not belonging to the category of interest; 3) False Positives (FP): number of cases incorrectly predicted as belonging to the category of interest; and 4) False Negatives (FN): number of cases incorrectly predicted as not belonging to the category. TP $+\mathrm{FN}$ equal all positive cases $(\mathrm{P})$, and $\mathrm{TN}+\mathrm{FP}$ equal all negative cases $(\mathrm{N})$ in the sample. In this context, Accuracy is an overall measure of how the algorithm performed both on correctly predicting $\mathrm{TP}$ and TN. The version for imbalanced data is presented as well ${ }^{2}$. Recall measures the proportion of TP that were correctly predicted in relation to all positives $(\mathrm{TP}+\mathrm{FN})^{3}$. Precision measures TP as a proportion of all predicted elements $(\mathrm{TP}+\mathrm{FP})^{4}$. F-scores are

\footnotetext{
${ }^{2}$ Accuracy $=\frac{T P+T N}{T P+T N+F P+F N} ; \quad$ Balanced accuracy $=\frac{T P}{T P+F N}+\frac{T N}{T N+F P} / 2$

${ }^{3}$ Recall $=\frac{T P}{T P+F N}$

${ }^{4}$ Precision $=\frac{T P}{T P+F P}$
} 
overall classification assessments for the positive class (TP $+\mathrm{FN})$, and are calculated as the harmonic mean of precision and recall. Different betas give more prominence to recall or precision $^{5}$. Kappa is a measure of interrater reliability ${ }^{6}$. We used it here to measure the extent of agreement between each algorithm and human classification.

In addition to discipline subjects and sustainable development, we classified the papers according to different geographical categories: countries, regions, and income groups. For this, the World Bank's classification of countries was used. As the IEEE database's countries are not normalized, it was necessary to identify and extract country names from the affiliations field at two fronts: first, identifying mentions of country names and well-known cities in the author affiliations field. The remaining papers for which there was an affiliation field were identified by leveraging the first classification's knowledge base, querying fulltext indices to return similar records to those already classified. This approach provided an accuracy of classification of $97 \%$ in a random sample of 1,000 records checked manually. 17,555 out of 220,147 articles did not have an affiliation field, or it was not possible to identify their countries of affiliation.

Analysis

To explore AI documents with a potential contribution to sustainable development, we performed three analyses: The first one compares the number of documents by IEEE taxonomy categories and documents within those categories related to sustainable development. This intends to answer our first research question: To what extent is sustainable development addressed in AI research produced globally?

The second analysis responds to question 2, Which subject areas of AI show an emerging interest in sustainable development? It identifies those IEEE categories that have a critical growth rate in three aspects: the number of documents produced, estimated as:

$$
\text { 1) } C A G R_{P u b}=\left(\left[\sum_{i=1}^{19} \frac{P_{i}}{P_{2000}}\right]^{\left(\frac{1}{19}\right)}-1\right) * 100
$$

citation impact, estimated as:

inventive impact, estimated as:

$$
\text { (2) } C A G R_{C i t}=\left(\left[\sum_{i=1}^{17} \frac{C_{i}}{C_{2000}}\right]^{\left(\frac{1}{17}\right)}-1\right) * 100
$$

(3) $C A G R_{\text {PatCit }}=\left(\left[\sum_{i=1}^{17} \frac{P C_{i}}{P C_{2000}}\right]^{\left(\frac{1}{17}\right)}-1\right) * 100$

\footnotetext{
${ }^{5} F_{\beta}=\left(1+\beta^{2}\right) \frac{\text { Precision } * \text { Recall }}{\left(\beta^{2} * \text { Precision }\right)+\text { recall }}$

${ }^{6}$ In the terms of this study, kappa can be described as $k=\frac{2 *(T P * T N-F N * F P)}{(T P+F P) *(F P+T N)+(T P+F N) *(F N+T N)}$
} 
Where $C A G R=$ Compound Annual Growth Rate; $P u b=$ number of documents; $C i t=$ citations; PatCit $=$ patent citations; $P_{i}=$ number of documents in year $i$ of the time series $(1$ $=$ year 2000, and $19=$ year 2019), $C_{i}$ is the number of citations in year $i(1=$ year 2000, and $17=$ year 2017); and $P C_{i}$ is the number of patent citations in year $i(1=$ year 2000, and $17=$ year 2017). Concerning the citation indicators, we take as the most recent year 2017 because of citation lags that may show a decrease at the end of the series that is not accurate (van Raan 2005). Additionally, we calculated a composite indicator based on the average ranking of the three variables. A comparison between sustainable development-related production and other production is offered for those areas within the main subject of Computational and Artificial Intelligence.

In addition, we performed a third analysis to address the research question to what extent does AI stimulate collaboration between regions of the world? Given that there is competition among nations for leadership in this field (Mou 2019), it can be expected that cross-regional collaboration is minimum, as well as collaboration between countries classified in different income groups. We also offer a comparison between economic regions in terms of the World Bank's income categories (World Bank 2021). The indicators for performing these comparisons rely on counting papers once for each author/region. It produces the same number of total papers if there is no collaboration (authors of the papers belong to the same region). Otherwise, the number could be greater than the number of papers, given that collaboration from two authors of different regions could count as 1 for each region. The indicators are as follows:

1) Rate of interregional collaboration $=, \sum \frac{P_{r}}{P}$, where $P_{r}=$ number of publications produced by at least one co-author from region $r$ and $P=$ total number of documents.

2) Rate of cross-income collaboration $=\sum \frac{P_{i c}}{P}$, where $P_{i c}=$ number of publications produced by at least one co-author from income category $i c$ and $P=$ total number of documents.

Finally, we performed a social network analysis of countries to identify the most central nodes through co-authorships. The literature on research collaboration at the country level is consistent in the finding that a few countries concentrate a tremendous amount of power in the networks, while many others stay at the periphery of collaboration (Kwiek 2020; King 2011; Wagner 2008). This gives those central countries the power to control knowledge flows and the rules of collaboration (Olechnicka et al. 2019). We explored the collaboration structure of AI papers related to SD by analyzing their betweenness centrality, which refers to the notion that "a point in a communication network is central to the extent that it falls on the shortest path between pairs of other points" (Freeman, 1977, p.35), an idea that was first proposed by Bavelas (1948). The higher the betweenness centrality of a node, the more power to transmit or retain information, which may yield it a greater control over the flow of information across the network. To compute betweenness centrality we used the Gephi software implementation, which produces an indicator for each node between 0 -lowest betweenness centrality- and 1 -highest-. Gephi allows to use of weighted networks and its betweenness centrality algorithm is based on the Brandes (2001) algorithm (Gephi 2021). 


\section{Results}

Consideration of sustainable development in AI research

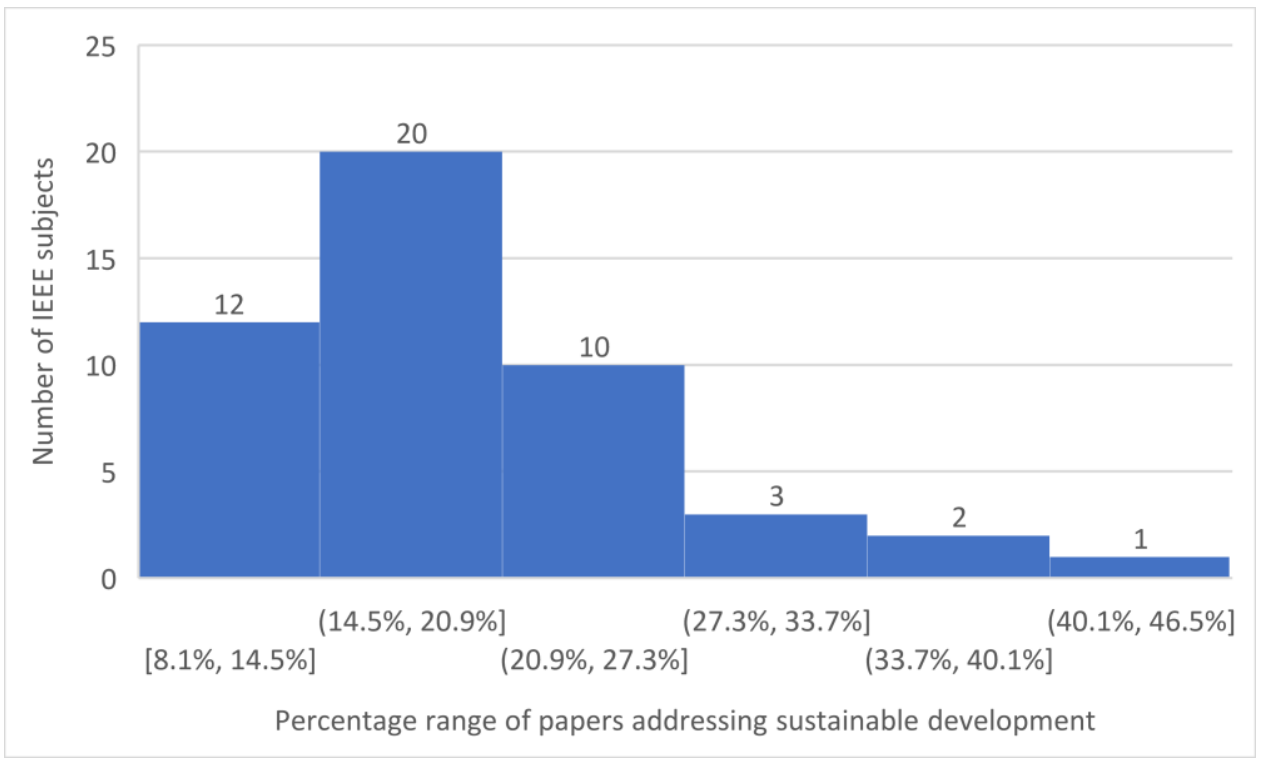

Figure 1. Distribution of the number of subjects by the percentage of documents addressing sustainable development.

Source: Authors' elaboration based on IEEE Xplore and taxonomy. The size of the intervals was automatically chosen by the software

Figure 1 shows the number of documents by IEEE taxonomy top-level subject and the percentage of publications that address sustainable development. In total, 197,384 documents had an IEEE taxonomy classification. Of these, 44,563 papers were classified as sustainable development, which means that approximately $23 \%$ of papers in our dataset addressed sustainable development, based on our keyword identification. Figure 1 shows a skewed distribution of these categories. Most of the subjects address sustainable development between $14 \%$ and $27 \%$. Only six subjects address sustainable development in more than $27 \%$ of their papers, with only one reaching the limit of $40 \%$. This hints that sustainable development is a subject highly concentrated in specific domains of AI.

To find which domains within the core categories of AI concentrate research on sustainable development, we analyzed the IEEE subject Computational and Artificial Intelligence. Figure 2 shows a mapping exercise in which we show the main subcategories, their different subjects, and the extent to which they address sustainable development (the percentage is relative to the subcategory). The figure shows that many subcategories of Artificial Intelligence address SDGs from 20 to 25\%. In contrast, most subcategories in Neural Networks, Computational Intelligence, and Logic do not reach even $20 \%$. 

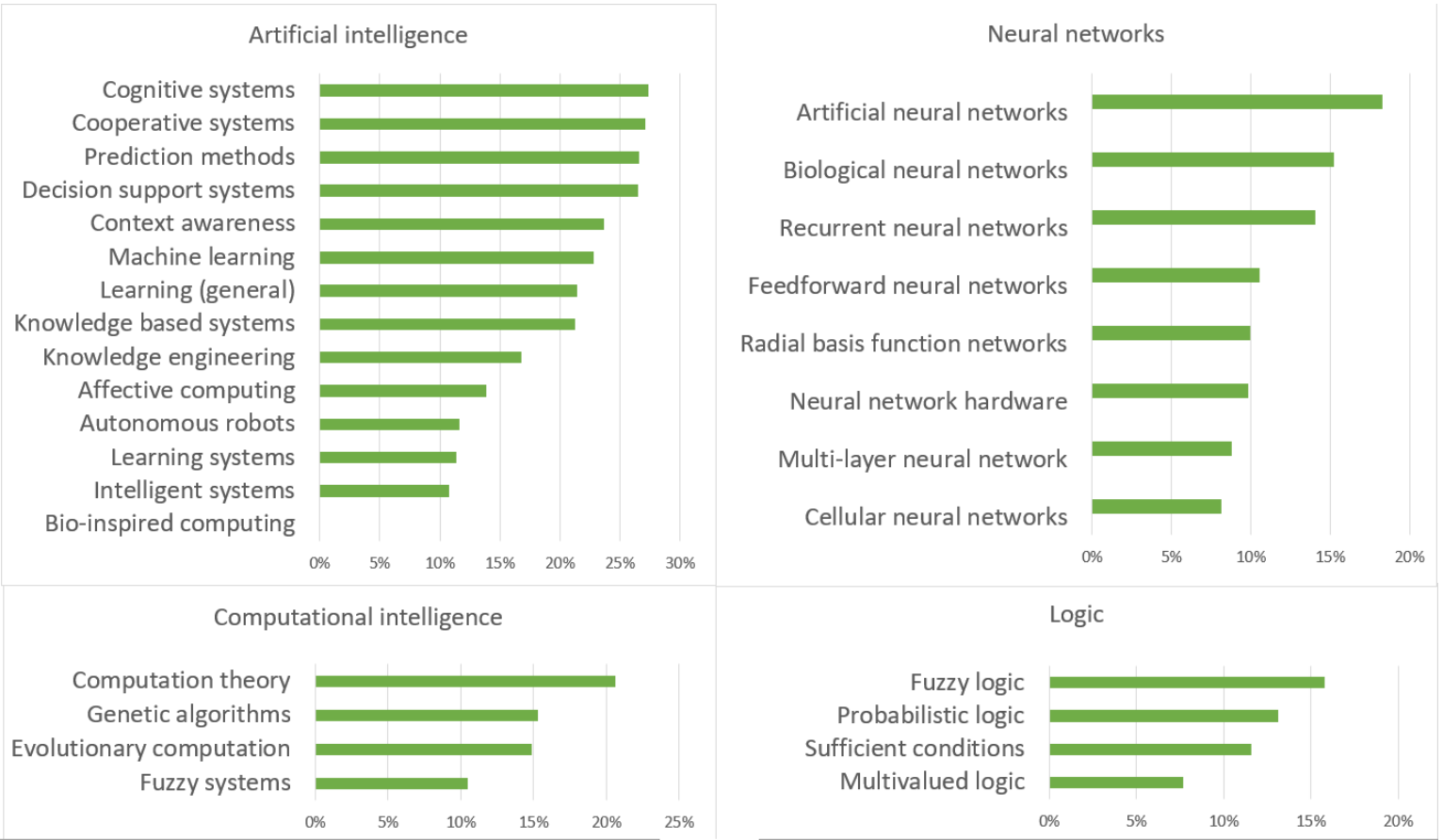

Figure 2. Percentage of papers within the Computational and Artificial Intelligence category that address SDGs, classified into Artificial Intelligence, Neural Networks, Computational Intelligence, and Logic.

\section{IEEE subjects with an emerging interest in sustainable development.}

The indicators for total AI documents, AI documents related to sustainable development, and AI documents not classified as sustainable development are in Table 4. The calculations include CAGR production, scientific (based on citations from bibliographic outputs), and invention impact (based on patents). From the data, we find that sustainable development documents supersede other AI documents in production growth and scientific impact. This shows that papers in AI are slightly increasingly addressing sustainable development subjects and that the citation rate to articles addressing sustainable development is increasing above-average citation growth. However, sustainable development documents perform below average on inventive impact as measured by patent citations (CAGR).

Table 4. Production, scientific impact, and inventive impact indicators for AI papers for the analyzed database.

\begin{tabular}{|c|c|c|c|}
\hline & $\begin{array}{l}\text { Production } \\
\text { (CAGR) }\end{array}$ & $\begin{array}{l}\text { Scientific Impact } \\
\text { (CAGR) }\end{array}$ & $\begin{array}{l}\text { Inventive impact } \\
\text { (CAGR) }\end{array}$ \\
\hline All docs & $25 \%$ & $25 \%$ & $18.3 \%$ \\
\hline SD docs & $31 \%$ & $32 \%$ & $17.5 \%$ \\
\hline Non-SD docs & $24 \%$ & $24 \%$ & $18.4 \%$ \\
\hline
\end{tabular}

Note: All docs refer to the totality of AI documents; SD docs point to AI documents related to sustainable development; non-SD docs relate to AI documents not classified as related to sustainable development.

Source: own calculations based on IEEE Xplore and taxonomy

The above indicators allowed us to derive a ranking of emerging areas in AI and sustainable development. The ranking was calculated as the average of the rankings in the three above indicators for each IEEE subject area - all 52 engineering categories. The ranking identifies 
the subjects that show a more pronounced growth in the number of documents and citations in bibliographic outputs, and a higher score in patent cites per document. Table 5 shows the top 5 general IEEE engineering subjects.

Table 5. Top 5 emerging subjects addressing sustainable development in AI papers

\begin{tabular}{|c|c|c|c|c|c|c|c|}
\hline IEEE subject & $\begin{array}{l}\text { CAGR } \\
\text { Prod } \%\end{array}$ & $\begin{array}{l}\text { CAGR } \\
\text { Cit. } \%\end{array}$ & $\begin{array}{l}\text { CAG } \\
\text { R Pat } \\
\text { Cit. } \\
\%\end{array}$ & $\begin{array}{l}\text { Rank } \\
\text { Prod. }\end{array}$ & $\begin{array}{l}\text { Rank } \\
\text { Cit. }\end{array}$ & $\begin{array}{l}\text { Rank } \\
\text { Pat }\end{array}$ & $\begin{array}{l}\text { Avg } \\
\text { rank }\end{array}$ \\
\hline $\begin{array}{l}\text { Ultrasonics, ferroelectrics, and } \\
\text { frequency control }\end{array}$ & 34.2 & 36.1 & 29.1 & 2 & 7 & 3 & 4 \\
\hline Education & 38.3 & 59.8 & 27.3 & 1 & 2 & 11 & 4.7 \\
\hline Consumer electronics & 33.2 & 43.6 & 27.6 & 4 & 3 & 9 & 5.3 \\
\hline Electrical engineering & 33.3 & 40.7 & 23.0 & 3 & 4 & 17 & 8 \\
\hline $\begin{array}{l}\text { Electromagnetic compatibility } \\
\text { and interference }\end{array}$ & 27.8 & 65.0 & 36.4 & 29 & 1 & 2 & 10.7 \\
\hline
\end{tabular}

Note: CAGR stands for Cumulative Aggregate Growth Rate. Prod stands for documents, Cit. stands for the sum of citations, Pat ind refers to patent citations per paper. Rankings with lower values must be understood as being at the top of the list. Each ranking refers to the ordering of the values of CAGR and Pat ind. The average ranking is the mean of the three rankings in the columns.

Table 6 shows the correlation coefficients between the three rankings. The correlations suggest that production growth is moderately and positively associated with scientific impact as measured by citation growth. However, inventive impact as measured by patent citations growth is weakly related to production and moderately related to scientific impact. This means that even if sustainable development papers increase their production and citation growth, it does not necessarily indicate that sustainable development-related knowledge will make its way into the development of technologies.

Table 6. Overall correlation coefficients between the ranking of production, the ranking of scientific impact, and the ranking of inventive impact.

\begin{tabular}{|c|c|c|c|}
\hline & $\begin{array}{l}\text { Production } \\
\text { Ranking }\end{array}$ & $\begin{array}{l}\text { Scientific } \\
\text { impact } \\
\text { ranking }\end{array}$ & $\begin{array}{l}\text { Inventive } \\
\text { impact } \\
\text { ranking }\end{array}$ \\
\hline Production Ranking & 1 & 0.6 & 0.4 \\
\hline Scientific impact ranking & & 1 & 0.5 \\
\hline Inventive impact ranking & & & 1 \\
\hline
\end{tabular}

Similarly, we explored more in-depth the subjects strictly related to the category Computational and Artificial intelligence. This category is composed of artificial intelligence, computational intelligence, logic, machine intelligence, and neural networks. Table 7 shows the top 5 subjects across these categories: 
Table 7. Top 5 emerging subjects addressing sustainable development in AI papers within the category Computational and Artificial Intelligence

\begin{tabular}{lrrrrrrr}
\hline IEEE subject & $\begin{array}{l}\text { CAGR } \\
\text { Prod } \%\end{array}$ & $\begin{array}{l}\text { CAGR } \\
\text { Cit. \% }\end{array}$ & $\begin{array}{l}\text { CAGR Pat } \\
\text { Cit } \%\end{array}$ & $\begin{array}{l}\text { Rank } \\
\text { Prod. }\end{array}$ & $\begin{array}{l}\text { Rank } \\
\text { Cit. }\end{array}$ & $\begin{array}{l}\text { Rank } \\
\text { Pat }\end{array}$ & $\begin{array}{l}\text { Avg } \\
\text { rank }\end{array}$ \\
\hline Prediction methods & 36.4 & 55.6 & 34.9 & 2 & 1 & 1 & 1.3 \\
Computation theory & 36.3 & 38.5 & 27.4 & 3 & 5 & 5 & 4.3 \\
Machine learning & 39.3 & 31.3 & 31.9 & 1 & 11 & 3 & 5.0 \\
Learning (artificial & 35.2 & 43.7 & 21.9 & 4 & 3 & 9 & 5.3 \\
$\begin{array}{l}\text { intelligence) } \\
\text { Biological neural networks }\end{array}$ & 28.9 & 34.8 & 21.7 & 10 & 9 & 10 & 9.7 \\
\hline
\end{tabular}

Note: CAGR stands for Cumulative Aggregate Growth Rate. Prod stands for documents, Cit. stands for the sum of citations, Pat ind refers to patent citations per paper. Rankings with lower values must be understood as being at the top of the list. Each ranking refers to the ordering of the values of CAGR and Pat ind. The average ranking is the mean of the three rankings in the columns. Learning (artificial intelligence) is differentiated from Machine learning and Learning systems in the IEEE taxonomy. It refers specifically to AI applied to distance learning and e-learning.

Table 8 shows the correlation coefficients between the three rankings above. In the specific case of Computational and Artificial Intelligence, the correlations show that production growth is moderately and positively associated with scientific impact as measured by citation growth. Inventive impact is strongly related to production, which suggests that for this set of technologies an increase in the number of papers is accompanied by their incorporation into technologies. A more moderate association is found between inventive impact and scientific impact, which may indicate that the industry does not necessarily incorporate highly regarded academic contributions.

Table 8. Correlation coefficients for the subject Artificial and Computational Intelligence

\begin{tabular}{|c|c|c|c|}
\hline & $\begin{array}{l}\text { Production } \\
\text { Ranking }\end{array}$ & $\begin{array}{l}\text { Scientific } \\
\text { impact } \\
\text { ranking }\end{array}$ & $\begin{array}{l}\text { Inventive } \\
\text { impact } \\
\text { ranking }\end{array}$ \\
\hline$\overline{\text { Production Ranking }}$ & 1 & 0.6 & 0.8 \\
\hline Scientific impact ranking & & 1 & 0.5 \\
\hline Inventive impact ranking & & & 1 \\
\hline
\end{tabular}

Collaboration in AI papers related to sustainability between regions, income groups, and countries.

Production of AI papers indexed in IEEE Xplore is highly concentrated in high-income countries from the East Asia \& the Pacific, Europe \& Central Asia, and North America regions. Tables 8 and 9 show the distribution of papers by region and income group. In table 8 , we observe that East Asia \& the Pacific concentrate most of the production on AI and AI related to SD in absolute terms. However, in relative terms, it is also the region with the lowest share of papers associated with SD (19\%) as compared to their total AI production. In contrast, Sub-Saharan Africa exhibits the lowest production of total AI papers and papers related to $\mathrm{SD}$, but also the highest share of papers on SD (31\%) as compared to their total 
AI production. The table suggests that as income decreases, total production of documents on AI decreases too, but the share of documents on AI related to SD increases. Table 9 supports this observation: production in absolute terms decreases as income decreases, and the share of SD-related production increases as income decreases. This suggests a negative relationship between income and the share of SD-related papers.

Table 8. Number of AI documents by region

\begin{tabular}{lrrr}
\hline Region & \multicolumn{1}{c}{ Docs } & Docs - SD & Docs - non-SD \\
\hline East Asia \& Pacific & 100,692 & 18,694 & 81,998 \\
Europe \& Central Asia & 56,188 & 11,771 & 44,417 \\
North America & 47,738 & 9,990 & 37,748 \\
South Asia & 16,469 & 4,808 & 11,661 \\
Middle East \& North Africa & 10,191 & 2,587 & 7,604 \\
Latin America \& Caribbean & 7,339 & 1,687 & 5,652 \\
Sub-Saharan Africa & 1,481 & 461 & 1,020 \\
\hline
\end{tabular}

Note: Docs refers to the total number of AI documents; Docs - SD relates to the number of documents related to sustainable development; Docs - non-SD relates to the number of documents not identified as related to sustainable development.

Table 9. Number of AI documents by income group

\begin{tabular}{lrrc}
\hline Income group & Docs & Docs - SD & Docs - non-SD \\
\hline High income & 119,397 & 24,263 & 95,134 \\
Upper middle income & 95,163 & 18,355 & 76,808 \\
Lower middle income & 21,173 & 6,077 & 15,096 \\
Low income & 203 & 64 & 139 \\
\hline
\end{tabular}

Note: Docs refers to the total number of AI documents; Docs - SD refers to the number of documents related to sustainable development; Docs - non-SD refers to the number of documents not identified as related to sustainable development.

In general, inter-regional collaboration in AI papers between countries is low. The ratio between the total number of papers and co-authorships between regions is 1.18. Papers related to sustainable development have a lower ratio of 1.12. Papers not identified as related to sustainable development have a ratio of 1.18. This means that most papers involve only collaboration within their region.

In terms of income group, the data shows similar patterns. The ratio between the total number of papers with a country of affiliation identified and co-authorships between income groups is 1.16. Papers related to sustainable development and papers not related to sustainable development have the same ratio. As in the regional case, this means that most papers involve only collaboration within their income group.

Figure 3 shows the social network graph of country co-authorships for SD-related papers. Node size and color intensity indicate the betweenness centrality of countries, and link thickness represents the number of co-authorships between countries. The top 10 percentile by betweenness centrality is composed, in descending order, by the United States, France, China, United Kingdom, India, Spain, Japan, Austria, Saudi Arabia, Germany, Malaysia, Australia, Portugal, Croatia, and Russia. Of these fifteen countries, eleven are high-income countries, three are upper-middle income, and one (India) is classified as a low-income country (HICs) - (World Bank 2021). Although the top 10 percentile is dominated by HICs, the general correlation between betweenness centrality and income group is only 0.21 , which shows that there is a diversity of countries around the most central ones. The social network analysis partly confirms the concentration of power in a few countries pointed out by the 
literature on collaboration and shows the emergence of new powers in SD-related AI research.

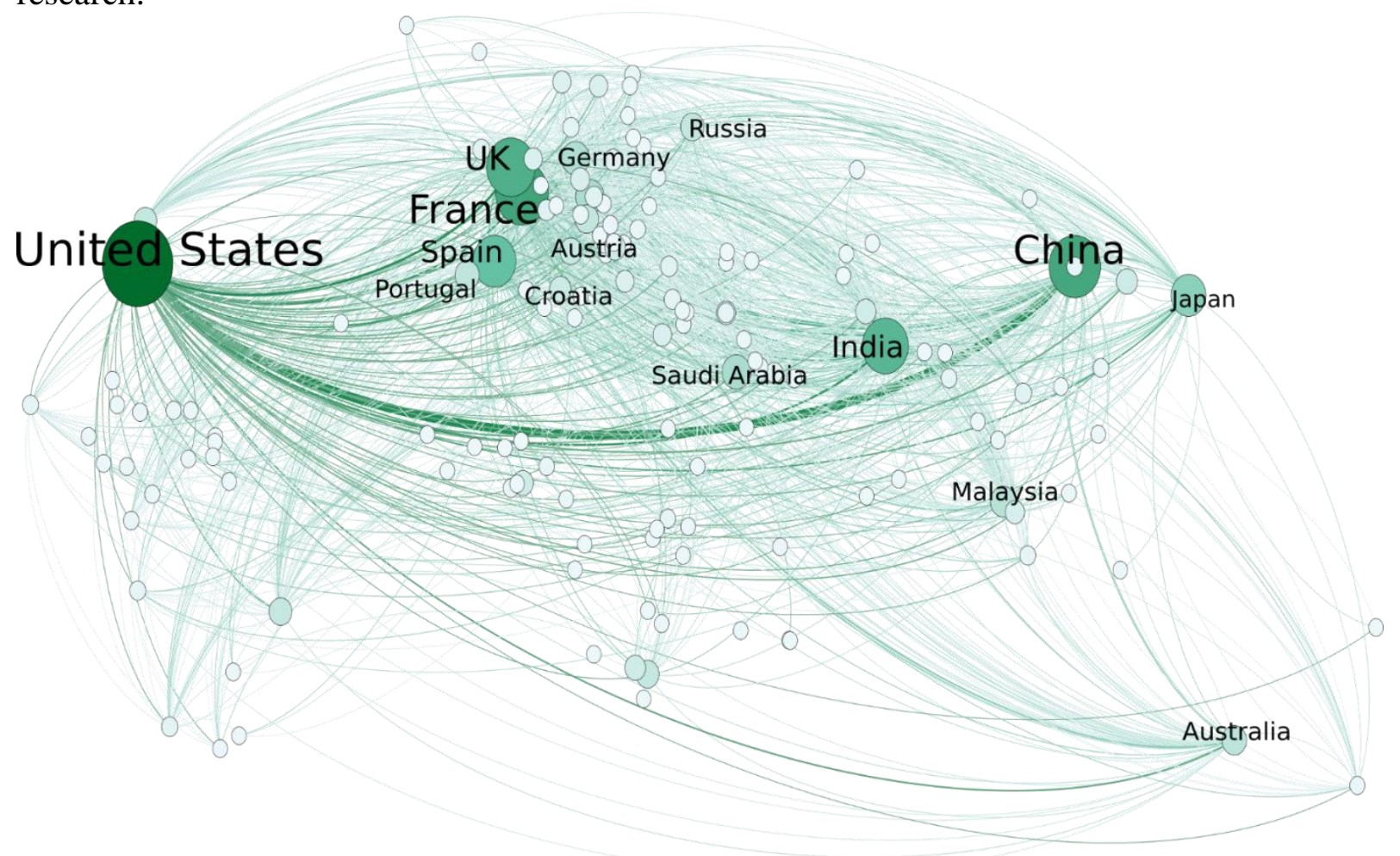

Figure 3. Co-authorship network of countries*

* Nodes positions attempt to preserve the location of countries in the Mercator projection of the world map. Size and color indicate betweenness centrality; only countries in the top 10 percentile by betweenness centrality are shown for visualization.

\section{Discussion and conclusion}

In this research, we addressed the questions: 1) To what extent is sustainable development addressed in AI research produced globally? 2) Which subject areas of AI show an emerging interest in sustainable development? And 3) To what extent does AI stimulate collaboration between regions of the world?

We found that AI papers address sustainable development to a limited extent. In most IEEE engineering subjects, the percentage of papers addressing SD ranges between $8 \%$ and $30 \%$. The most related ones are Power engineering and energy, Geoscience and remote sensing, Engineering management, and Engineering in medicine and biology. Even in these IEEE subjects, we only found between $30 \%$ and $40 \%$ of papers related to SD. We found similar results when analyzing the IEEE subject Computational and Artificial Intelligence in detail. The small number of explicit references to SD in AI papers indicates that discussions on the use of AI for grand challenges such as poverty reduction, inequality, peace, among others, are not central to the engineering AI literature.

The above finding is exploratory because it relies on a keyword-based identification of SDGs, and Armitage et al. (2020) have shown that there are two main risks when performing this classification task: overestimation and underestimation. To account for this, we tested different algorithms and chose the one that fit closer to a sample of human-classified papers. Still, we understand that there is ample room for new methods, such as including forward and backward citations (Bautista-Puig et al. 2021) and relating SDG-keywords to interdisciplinary clusters (Ràfols et al. 2021). In the case of an overestimation, our conclusion that sustainable development is addressed to a limited extent in our sample would 
be further strengthened. The possibility of an underestimation exists, but we consider that the differences in growth trends between total, SD-related, and non-SD indicators show that the sets are sufficiently similar within classes and differ between them. This provides a level of confidence in our findings. We, therefore, consider that motivating engineers to break their silos and explicitly reflect on the connection between their technologies and society will help increase the value of AI research beyond technical progress and efficiency gains.

Global AI research policies could build on our finding that SD is an emerging concern for engineering researchers in certain IEEE subjects such as Ultrasonics, ferroelectrics, frequency control, Education, Consumer Electronics, Electrical Engineering, and Electromagnetic Compatibility and Interference. Understanding in which ways AI is being linked to SD by engineers in these and other fields could help design strategies to make AI more relevant for grand societal challenges. Engineering education is one key field in which interdisciplinary research about sustainable development could have a profound impact, given the cross-cutting nature of education to the development of all engineering fields.

Furthermore, we have highlighted more specific areas of AI in which there is an emerging interest in SDGs. These are: Prediction methods, Computation Theory, Machine learning, learning (applied to e-learning and distance learning), and Biological neural networks. Some of the specific issues addressed by these papers include early prediction of diseases and diagnostics, health monitoring, health management and decision making, electricity demand, disaster risk reduction, water pollution detection, geological conditions to ensure water supply, pest control, domain ontologies from text for education, intelligent educational environment, modeling the visual cortex, understanding animal learning, among others. Therefore, the potential of AI for sustainable development is huge if properly fostered through connections with public policies and higher education. Our use of a composite indicator helped us to identify IEEE subjects with a distinctive combined growth in production, scientific impact, and inventive impact. We understand this is but one way in which promising IEEE subjects can be identified, and depending on the needs of funding agencies and research organizations different indicators can be used. However, the examples we have found and described above give some confidence that ours is one suitable approach. In terms of collaboration, our findings show that inter-regional and inter-income group collaboration is not very frequent. The data shows that 1 in 7 papers will produce an interregional or inter-income group collaboration. This pattern may be a reproduction in research of the competitive environment for leading technological development, as pointed out by the literature (Mou 2019; Sørensen et al. 2016). We also see an enormous concentration of research capacity in East Asia \& Pacific, Europe \& Central Asia, and North America, which shows a gap in knowledge production that confirms the technological and knowledge dependence of certain regions and reinforces the global division between centers and peripheries (Kshetri 2020). We think that a competitive and monopolistic environment is likely to produce an even greater gap between technologically advanced countries and the rest, opposite to the stated goals of global AI policies and SD (OECD 2020; IEEE 2019) ${ }^{7}$. Based on our experience in policymaking in an upper middle-income country, we know that the technological gap will not be bridged if countries at the periphery continue to be seen as mere buyers of technology and their governments continue to accept this role. As we showed, lower income countries produce a higher share of AI research related to SD in comparison to their total production than higher income countries, which indicates that there is an opportunity to build on this interest and research capacity to make AI work for SD. If global AI policies are to support SD and its global agenda, then there should be a global 
commitment to improve AI capacity in those countries that remain peripheral, instead of concentrating power and capacity on a few ones. This requires a change towards a more generous attitude in international relationships that is not supported by the current climate of competition between countries such as the USA, Russia, and China, and by the current rate of natural resources extraction.

An interesting question, posed by an anonymous reviewer, is if collaboration in terms of the number of coauthors differs for SDG-related and non-SDG research. A significant difference could show a changing pattern in specific subjects, which may provide a hint that SDGrelated research in AI could be changing the structure of scientific collaboration. Although this is not the focus of this paper, we performed an exploratory analysis of the number of authors for both the aggregated sample and each IEEE subject. We found that the average number of authors is very similar for SDG-related papers (3.5 authors) and non-SDG papers (3.7). In terms of IEEE subjects, we found a similar pattern. Based on these observations we considered that the number of authors does not produce new information to what we have already found through the network analysis. Therefore, we did not include this as part of the results. However, these exploratory results support our finding that researching on SDGs does not seem to produce novel collaboration patterns, which strengthens our observation that there is a risk that SDGs in AI become a buzzword with a small effect on the transformation of traditional scientific collaboration patterns.

The above findings are relevant for research policy and for citizen engagement. Increasing awareness of researchers about SD and their motivation to produce socially relevant research, as well as increasing the capacity to produce such research, is determinant for solving some of the grand challenges of the world. More aware and motivated researchers will understand the need to collaborate with stakeholders beyond academia to produce technologies that matter for their societies while developing new technical and social skills that will build the capacity of countries to determine and pursue their own research agendas. This is aligned with the stated aims of multilateral organizations such as the OECD and the European Union, as well as institutions such as the IEEE, which have expressed a determined intention to produce AI research for the benefit of the economy, the environment, and society. They have produced formal statements to support this orientation of AI to pave the way to that goal. Our results offer an empirical basis for these organizations and for countries to connect AI engineering research with society. Sustainable development provides a framework to achieve this connection, which can be compared to what Jane Goodall calls a connection between our brain -committed to advancing the frontier of science- and our heart, which connects scientific advancement to the planet's well-being.

\section{Future research}

This is an exploratory analysis that opens different avenues of research, for instance in deepening our analysis on collaboration, interdisciplinarity, and emerging subjects. Further research should also investigate the social, economic, and environmental contextual dimensions of the countries that produce AI research. On the technical side, our research can be enhanced by extending the analysis of AI through different classification standards beyond IEEE and including discussions of Artificial Intelligence interpretations as discipline and as a research field. Documenting the invention of AI technologies for SD through social construction between academia, citizens, governments, and entrepreneurs, will help derive policy-relevant lessons and strengthen the inclusion of citizens' concerns on AI.

\section{Declarations}


Funding: This work was supported by the Ministry of Science, Technology, and Innovation of Colombia (MinCiencias) under Grant 848-2019 and the Colombian Society of Engineering Physics (SCIF), Colombia.

Conflicts of interest: the authors declare no conflict of interests to the best of their knowledge.

Availability of data and material: upon request, we can make available our database, but this cannot be disclosed publicly because of IEEE's API terms and conditions.

Authors' contributions: The authors contributed equally to this work.

\section{References}

Armitage, C. S., Lorenz, M., \& Mikki, S. (2020). Mapping scholarly publications related to the Sustainable Development Goals: Do independent bibliometric approaches get the same results? Quantitative Science Studies, 1(3), 1092-1108.

Bavelas, Alex. (1948). A mathematical model for group structures. Applied Anthropology 7(3), 1630.

Brandes, U. (2001). A faster algorithm for betweenness centrality. Journal of mathematical sociology, 25(2), 163-177.

Bautista-Puig, N., Aleixo, A. M., Leal, S., Azeiteiro, U., \& Costas, R. (2021). Unveiling the Research Landscape of Sustainable Development Goals and Their Inclusion in Higher Education Institutions and Research Centers: Major Trends in 2000-2017. Frontiers in Sustainability, 2, 620743. https://doi.org/10.3389/frsus.2021.620743

Breschi, s., Lissoni, F., Montobbio, F. (2005). From publishing to patenting. Do productive scientists turn into academic inventors? Revue d'économie industrielle, (110), 75-102

Cassi, L., Lahatte, A., Rafols, I., Sautier, P., \& De Turckheim, E. (2017). Improving fitness: Mapping research priorities against societal needs on obesity. Journal of Informetrics, 11(4), 1095-1113.

Chavarro, D., Perez-Taborda, J. A., \& Avila, A. (2021). Connecting Brain and Heart: Artificial Intelligence for Sustainable Development. In 18th International conference on scientometrics and informetrics ISSI 2021 (pp. 229-240). ISSI.

Chavarro, D., Ràfols, I., \& Tang, P. (2018). To what extent is inclusion in the Web of Science an indicator of journal 'quality'?. Research Evaluation, 27(2), 106-118

Chavarro, D., Tang, P., \& Ràfols, I. (2017). Why researchers publish in non-mainstream journals: Training, knowledge bridging, and gap filling. Research policy, 46(9), 1666-1680.

Chavarro, D., Vélez, M. I., Tovar, G., Montenegro, I., Hernández, A., \& Olaya, A. (2017). Los Objetivos de Desarrollo Sostenible en Colombia y el aporte de la ciencia, la tecnología y la innovación. Colciencias. https://minciencias.gov.co/sites/default/files/ctei_y_ods__documento_de_trabajo.pdf

del Río Castro, G., Fernández, M. C. G., \& Colsa, Á. U. (2020). Unleashing the convergence amid digitalization and sustainability towards pursuing the Sustainable Development Goals (SDGs): A holistic review. Journal of Cleaner Production, 280(122204).

Dussel, E. D. (2018). Hacia la liberación científica y tecnológica en América Latina. Vectores de investigación, (14), 103-112.

European Commission. (2020). Emerging trends in STI policy. European Commission. https://stiplab.github.io/R2r/Emerging\%20trends\%20in\%20STI\%20policy.html

European Commission. (2021). "Proposal for a Regulation of the European Parliament and of the Council Laying down harmonised rules on artificial intelligence (Artificial Intelligence Act) and amending certain Union legislative acts". https://eur-

lex.europa.eu/resource.html?uri=cellar:e0649735-a372-11eb-9585-

01aa75ed71a1.0001.02/DOC_1\&format=PDF

European Parliament. (2020). Ethics of artificial intelligence. European Parliament. https://www.europarl.europa.eu/RegData/etudes/STUD/2020/634452/EPRS_STU(2020)634452 _EN.pdf

European Patent Office. (2020). Patents and the fourth industrial revolution. European Patent Office. 
http://documents.epo.org/projects/babylon/eponet.nsf/0/06E4D8F7A2D6C2E1C125863900517 B88/\$File/patents_and_the_fourth_industrial_revolution_study_2020_en.pdf

Elsevier (2020). Mapping research to advance the SDGs. Elsevier. https://www.elsevier.com/connect/sdg-report

Freeman, L. C. (1977). A Set of Measures of Centrality Based on Betweenness. Sociometry, 40(1), 35. https://doi.org/10.2307/3033543

Gardner, H. E. (2000). Intelligence reframed: Multiple intelligences for the 21st century. Hachette UK.

Gephi. (2021). GraphDistance (gephi-toolkit 0.9.2 API). https://gephi.org/gephitoolkit/0.9.2/apidocs/org/gephi/statistics/plugin/GraphDistance.html

Goodall, J. (2014). Interview. Brainpickings. https://www.brainpickings.org/2014/09/30/janegoodall-empathy/

Harari, Y. N. (2017). Reboot for the AI revolution. Nature News, 550(7676), 324.

IEEE. (2019). IEEE position statement on artificial intelligence. IEEE. https://globalpolicy.ieee.org/wp-content/uploads/2019/06/IEEE18029.pdf

IEEE. (2021a). IEEE Thesaurus. IEEE. https://www.ieee.org/content/dam/ieeeorg/ieee/web/org/pubs/ieee-thesaurus.pdf

IEEE. (2021b). Artificial Intelligence Systems (AIS) Related Standards. IEEE. https://standardsieee-org.ezproxy.utp.edu.co/initiatives/artificial-intelligence-systems/standards.html

Kagermann, H., Anderl, R., Gausemeier, J., Schuh, G., \& Wahlster, W. (Eds.). (2016). Industrie 4.0 in a Global Context: strategies for cooperating with international partners. Herbert Utz Verlag.

King, R. (2011). Power and Networks in Worldwide Knowledge Coordination: The Case of Global Science. Higher Education Policy 24(3), 359-76.

Klavans, R., Boyack, K. W., \& Murdick, D. A. (2020). A Novel Approach to Predicting Exceptional Growth in Research. ArXiv. arXiv:2004.13159.

Kshetri, N. (2020). Artificial Intelligence in Developing Countries. IEEE Annals of the History of Computing, 22(04), 63-68.

Kwiek, M. (2020). What large-scale publication and citation data tell us about international research collaboration in Europe: changing national patterns in global contexts. Studies in Higher Education, ahead of print, 1-21.

Mou, X. (2019). Artificial Intelligence: Investment Trends and Selected Industry Uses. World Bank. http://documents1.worldbank.org/curated/en/617511573040599056/pdf/ArtificialIntelligence-Investment-Trends-and-Selected-Industry-Uses.pdf

Navarro, J. (2018). The digital transformation imperative. An IDB science and business innovation agenda for the new industrial revolution. IDB. https://publications.iadb.org/en/digitaltransformation-imperative-idb-science-and-business-innovation-agenda-new-industrial

Nilson, N. (2010). The quest for artificial intelligence. A history of ideas and achievements. Cambridge University Press.

OECD. (2020). Principles of AI. OECD. http://www.oecd.org/going-digital/ai/principles/

Olechnicka, A., A. Ploszaj, \& D. Celinska-Janowicz (2019). The Geography of Scientific Collaboration. Routledge

OSDG. (2021). Home page. OSDG. https://osdg.ai/

Osoba, O. A., \& Welser, I. V. W. (2017). An intelligence in our image: The risks of bias and errors in artificial intelligence. Rand Corporation.

https://www.rand.org/pubs/research_reports/RR1744.html

Planes-Satorra, S. \& Paunov, C. (2019). The digital innovation policy landscape. OECD. https://doi.org/10.1787/6171f649-en

Powers, D. M. (2020). Evaluation: from precision, recall and F-measure to ROC, informedness, markedness and correlation. arXiv preprint arXiv:2010.16061.

Ràfols, I., Noyons, E., Confraria, H., \& Ciarli, T. (2021). Visualising plural mappings of science for Sustainable Development Goals (SDGs). ISSI 2021 Proceedings, 1, 949-955.

https://kuleuven.app.box.com/s/kdhn54ndlmwtil3s4aaxmotl9fv9s329

Russell, S. (2010). Artificial intelligence: a modern approach. Prentice Hall, 2010. 
Samoili, S., Lopez Cobo, M., Delipetrev, B., Martinez-Plumed, F., Gomez Gutierrez, E. and De Prato, G. (2021). AI Watch. Defining Artificial Intelligence 2.0. Publications Office of the European Union. DOI:10.2760/019901, JRC126426.

Schwab, K. (2017). The fourth industrial revolution. World Economic Forum.

Silver, D., Schrittwieser, J., Simonyan, K., Antonoglou, I., Huang, A., Guez, A., Hubert, T., Baker, L., Lai, M., Bolton, A., Chen, Y., Lillicrap, T., Hui, F., Sifre, L., van den Driessche, G., Graepel, T., \& Hassabis, D. (2017). Mastering the game of Go without human knowledge. Nature, 550(7676), 354-359. https://doi.org/10.1038/nature24270

Simmons, A. B., \& Chappell, S. G. (1988). Artificial intelligence-definition and practice. IEEE journal of oceanic engineering, 13(2), 14-42.

Sørensen, J., Tadayoni, R., \& Henten, A. (2016). 5G-Boundary Object or Battlefield? Communications \& Strategies, (102), 63-87.

UN. (2021). THE 17 GOALS / Sustainable Development. https://sdgs.un.org/goals

UNESCO. (2015). UNESCO science report: Towards 2030. UNESCO.

UNIDO. (2018). Industry 4.0 - The opportunities behind the challenges. Unido. https://www.unido.org/sites/default/files/files/2018-11/UNIDO GC17 Industry40.pdf

van Raan, A. F. (2005). For your citations only? Hot topics in bibliometric analysis. Measurement: interdisciplinary research and perspectives, 3(1), 50-62.

Wagner, C. (2008). The new invisible college. Science for development. Brookings Institution Press.

World Bank. (2021). World Bank Country and Lending Groups - World Bank Data Help Desk. https://datahelpdesk.worldbank.org/knowledgebase/articles/906519-world-bank-country-andlending-groups

Yamashita, I., Murakami, A., Cairns, S., \& Galindo-Rueda, F. (2021). Measuring the AI content of government-funded $R \& D$ projects: A proof of concept for the OECD Fundstat initiative (OECD Science, Technology, and Industry Working Papers No. 2021/09; OECD Science, Technology and Industry Working Papers, Vol. 2021/09). https://doi.org/10.1787/7b43b038en

Zellmer-Bruhn, M. E., Forbes, D. P., Sapienza, H. J., \& Borchert, P. S. (2021). Lab, Gig or Enterprise? How scientist-inventors form nascent startup teams. Journal of Business Venturing, 36(1), 106074. 